\title{
Neuromuscular Transmission in Diabetes: Response to High-Frequency Activation
}

\author{
Yitzhak Schillera and Rami Rahamimoff \\ Department of Physiology, Hebrew University Hadassah Medical School, Jerusalem 91010, Israel
}

In searching for the cellular correlates of diabetic neuropathy, we examined the response to tetanic stimulation of diabetic neuromuscular junctions and of age-matched controls. The experiments were performed on the soleus nerve muscle preparation of the rat in which diabetes was induced by streptozotocin. Tetanic potentiation was substantially lower in diabetic rats. In addition, it was found that the diabetic neuromuscular junction is more resistant to high-frequency stimulation than normal age-matched controls, in which such stimulation causes a progressively increasing number of failures in synaptic transmission. Tetanic failures cannot be predicted from the stochastic properties of transmitter release and are due to propagation block of action potentials into the nerve terminals. The resistance of diabetic nerves to tetanic stimulation is a function of the duration of diabetes; the earliest significant difference between the number of tetanic failures in diabetic and normal agematched controls was observed after $19 \mathrm{~d}$ of diabetes, and this difference grew with increased duration of diabetes. The resistance to tetanic stimulation in diabetic rats is reversed by insulin in vivo but not in vitro. Elevation in extracellular $\left[\mathrm{K}^{+}\right]$increases the number of tetanic failures in both diabetic and normal preparations. Furthermore, elevating extracellular $\left[\mathrm{K}^{+}\right]$to $\mathbf{8 . 5} \mathrm{mm}$ brings the number of tetanic failures into the range of tetanic failures in normal nerves. This finding is consistent with the hypothesis that differences in extracellular $\left[\mathrm{K}^{+}\right]$accumulation during high-frequency stimulation are responsible for the diabetic nerve's relative resistance to high-frequency stimulation. The lower number of failures corrects only partially the impaired neuromuscular transmission in the diabetic state, and there is an overall reduction in tetanic potentiation in diabetes.

Diabetic neuropathy is one of the most common complications of diabetes mellitus (Pirart, 1978; Brown and Asbury, 1984), affecting the sensory, motor, and autonomic peripheral nervous systems (Brown and Asbury, 1984; Thomas and Eliasson, 1984;

\footnotetext{
Received Oct. 31, 1988; revised Mar. 23, 1989; accepted Mar. 28, 1989.

We thank Dr. Simona Ginsburg for reading the manuscript and for helpful comments. The unfailing secretarial help of Mrs. Marsha Rapp and Ms. Yvonne Reich is greatly appreciated.

This work was supported by the Muscular Dystrophy Association, The National Institutes of Health (grant NS 17221-03), The Juvenile Diabetes Foundation, The Council for Tobacco Research, and the U.S.-Israel Binational Science Foundation. Equipment was purchased with the help of Sir Zelman Cowen Universities Fund.

Correspondence should be addressed to Rami Rahamimoff, Hebrew University Hadassah Medical School, P.O. Box 1172, Jerusalem, 91010, Israel.

- Present address: Department of Neurology, Hadassah University Hospital, Jerusalem, Israel.

Copyright (C) 1989 Society for Neuroscience $0270-6474 / 89 / 113709-11 \$ 02.00 / 0$
}

Harati, 1987). Although sensory diabetic neuropathy is the most troublesome, recent reports indicate that motor symptoms are almost as common (Brown and Asbury, 1984). Dyck et al. (1986) report that motor weakness in both arms and legs constitutes a common complaint among diabetic patients.

Electrophysiological studies show that several parameters are impaired by diabetes in the nerves of both patients and animal models. In the diabetic nerve the conduction velocity of the action potential was found to be reduced, the amplitudes of both motor- and sensory-evoked potentials were smaller, and the distal latency was prolonged (Mulder et al., 1961; Brown et al., 1980; Sima and Hay, 1981; Tackmann et al., 1981; Kennedy et al., 1982; Brown and Asbury, 1984; Dyck et al., 1985; Martinez, 1986; Harati, 1987). Despite the large amount of electrophysiological data accumulated, relatively little is known about cellular pathophysiology in diabetes (Brismar, 1983; Brismar et al., 1987). It was found that the synaptic transmission in diabetic rats is impaired, and both the quantal content and the amplitude of the end plate potential are significantly reduced after 3.5 months of experimental diabetes (Constantini et al., 1987). The pathogenesis of diabetic neuropathy is still by and large unknown, although several exciting suggestions have been made (Clements, 1979; Brown and Asbury, 1984; Greene et al., 1985, 1987; Low, 1987; Winegrad, 1987).

In this article we attempt to characterize one aspect of diabetic neuropathy at the rat neuromuscular junction. The nervous system usually varies the frequency of activation of a synapse in order to modify its transmission properties (for a review, see Erulkar, 1983). The aim of this study was therefore to comparc the response to tetanic stimulation of normal and diabetic nerve muscle preparations. Although the general conclusion of this study is that tetanic potentiation is significantly smaller in the diabetic neuromuscular junction, the analysis revealed that there are other differences, the main one being that the failures of synaptic transmission during high-frequency stimulation (Krnjevic and Miledi, 1958, 1959; Van Essen, 1973; Hatt and Smith, 1976; Spira et al., 1976; Grossman et al., 1979a; Smith, 1979, 1980; Kocsis et al., 1983) are fewer in diabetic preparations.

\section{Materials and Methods}

Animals and induction of diabetes. Male Sabra rats, bred at the Hebrew University Hadassah Medical School animal facility, were used in all experiments. Diabetes was induced in rats weighing $160-180 \mathrm{gm}$ by a single intraperitoneal injection of streptozotocin (STZ; Sigma) at a dose of $65 \mathrm{mg} / \mathrm{kg}$ (Byrne and Schein, 1981). Immediately prior to injection, STZ was dissolved in a normal Ringer's solution ( $\mathrm{pH} 4.5$ ) buffered with sodium citrate $(10 \mathrm{~mm})$. Control rats were injected with a similar volume of buffered Ringer's solution. Nonfasting glucose concentrations were determined $48-72 \mathrm{hr}$ after injection, and rats with glucose levels lower 

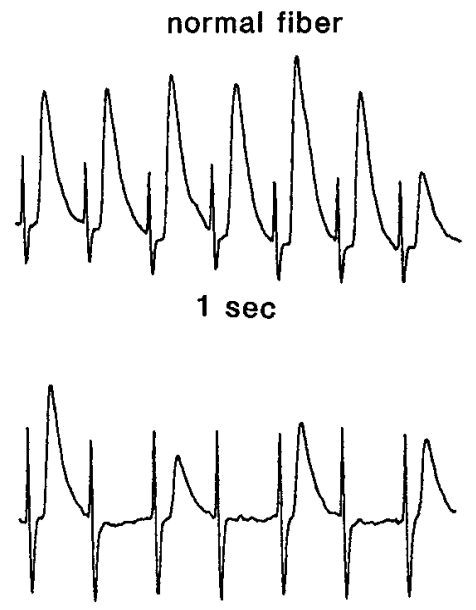

$15 \mathrm{sec}$

Figure 1. Tetanic failures in neuromuscular transmission: comparison between normal and diabetic fibers. Sweeps of intracellularly recorded e.p.p.s after 1,15 , and $35 \mathrm{sec}$ of a $100 \mathrm{~Hz}, 40$ sec nerve stimulation in a diabetic fiber (b, duration of diabetes $41 \mathrm{~d}$ ) and in an age-matched control fiber $(a)$. Note the progressively growing number of tetanic failures in the normal fiber compared with no tetanic failures in the diabetic fiber. b
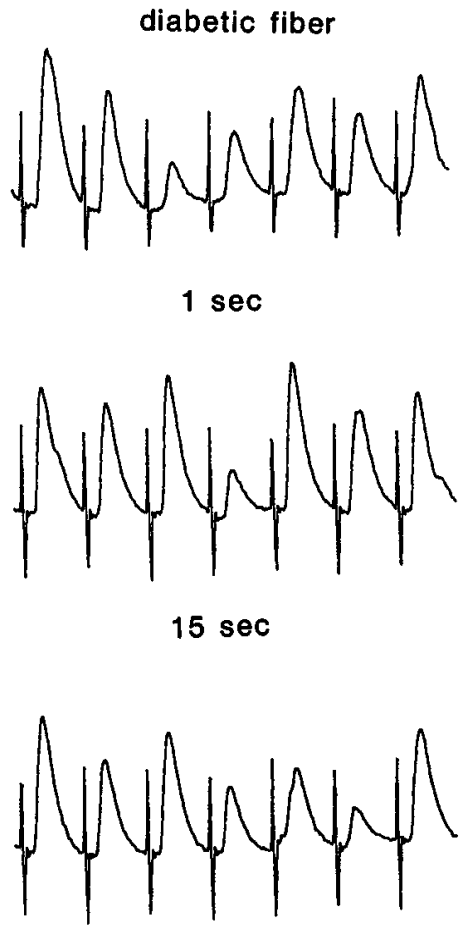

$35 \mathrm{sec}$ than $15 \mathrm{~mm}$ were discarded. After a period of $6 \mathrm{~d}-30$ weeks, the rats were fasted overnight, and glucose levels were again determined to assure the induction of diabetes; only rats with glucose levels above 15 mM were used for experimentation.

Rats receiving insulin treatment were given 2 daily injections (Insulin Leo NPH, Nordisk Gentofte). The exact dose of insulin was determined by daily measurements of blood glucose concentrations. The average dose of insulin given was $6-7 \mathrm{U} / \mathrm{d}$.

Electrophysiology. The experiments were performed on the soleus nerve muscle preparation and on the sciatic nerve. Both preparations were stimulated by a suction electrode with $60 \mathrm{msec}$ supramaximal square pulses (digital stimulator WPI 830 and isolator WPI 850A). Four different recording methods were employed:

1. Standard intracellular recordings from the postsynaptic membrane of the muscle, using glass microelectrodes filled with $3 \mathrm{M} \mathrm{KCl}$, with resistances of $8-15 \mathrm{M} \Omega$.

2. Standard extracellular recordings from the neuromuscular junction using fire-polished microelectrodes filled with normal Ringer's solution, with resistances of $2-4 \mathrm{M} \Omega$.

3. Subperineural recordings from the primary and secondary intramuscular branches innervating the soleus muscle, using glass microelectrodes filled with $1 \mathrm{M} \mathrm{NaCl}$ with resistances of 5-10 Mn.

4. Extracellular recordings from the sciatic nerve, proximal to the peroneal-anterior tibial bifurcation, using an additional suction electrode.

The electrical signals recorded via the microelectrodes were passed through a high-input-resistance amplifier (WPI 725), displayed on the screen of an oscilloscope (Tektronix 5113), and recorded on a tape recorder (HP 3964A). Later, the recorded electrical signals were either displayed on a chart recorder (Gould 2200S) or fed to a Digital computer (PDP MINC 11/23) and analyzed with special programs written for that purpose.

Solutions and materials. The standard bathing solution used was 134 $\mathrm{mm} \mathrm{NaCl}, 5 \mathrm{~mm} \mathrm{KCl}, 0.6 \mathrm{~mm} \mathrm{CaCl}_{2}, 5 \mathrm{mM} \mathrm{MgCl}_{2}, 15 \mathrm{~mm} \mathrm{NaHCO}_{3}$, $1 \mathrm{mM} \mathrm{NaH} \mathrm{PO}_{4}$, and $11 \mathrm{~mm}$ D-glucose. In some experiments, the concentration of the divalent ions was altered. In all experiments, the solutions were bubbled with a $95 \% \mathrm{O}_{2}-5 \% \mathrm{CO}_{2}$ mixture, and the $\mathrm{pH}$ was adjusted to 7.3-7.4.
Statistics. All results are displayed as means \pm SEM. Statistical analysis was performed using the 1-tailed Student $t$ test or the Mann-Whitney test.

\section{Results}

The purpose of this study was to compare the response of the normal and diabetic nerve muscle preparations to tetanic potentiation. Initial experiments had shown that not only were the properties of transmitter release altered by diabetes, but also the rate of success of synaptic activation. We therefore first describe this complicating phenomenon and then assess the overall damage to neuromuscular transmission by diabetes.

\section{Tetanic failures in normal and diabetic nerves}

Recordings of end plate potentials (e.p.p.) from the neuromuscular junction of normal rats during high-frequency stimulation show intermittent failures of synaptic transmission (tetanic failures). One example of this phenomenon is given in Figure $1 a$. The nerve innervating the soleus muscle of a 3-month-old normal rat was stimulated at a rate of $100 \mathrm{~Hz}$. During the first few seconds, each stimulus (marked by the stimulation artifact) resulted in an e.p.p. After several seconds of continuous stimulation, intermittent failures appeared, and there were stimuli that were not followed by an c.p.p. (for example, in the middle trace of Fig. 1a, 3 out of 7 e.p.p.s failed). As nerve stimulation continued, the number of tetanic failures increased, and in many instances their number exceeded that of "successful" e.p.p.s (in the lower trace of Fig. 1a, 5 out of 7 stimuli failed to induce an e.p.p.).

An example of high-frequency stimulation in a diabetic nerve 
is shown in Figure $1 b$. Again, the nerve was stimulated at a rate of $100 \mathrm{~Hz}$ for $40 \mathrm{sec}$. To our surprise, contrary to the results in the normal nerve, no tetanic failures appeared, and the diabetic nerve was relatively resistant to high-frequency stimulation.

The diabetic nerve fiber presented in Figure $1 b$ was not an exception. Figure 2 and Table 1 compare tetanic failures in diabetic nerves (duration of diabetes 28-30 weeks) to normal age-matched control nerves. Figure 2 plots the average number of "successful" e.p.p.s in $1 \mathrm{sec}(100$ minus the number of tetanic failures) as a function of the stimulation duration in a $60 \mathrm{sec}$, $100 \mathrm{~Hz}$ nerve stimulation. Table 1 presents the average number of e.p.p.s in $1 \mathrm{sec}$ during the last $5 \mathrm{sec}$ of a $40 \mathrm{sec}, 100 \mathrm{~Hz}$ nerve stimulation in diabetic and control rats. The data clearly demonstrate that diabetic nerves have a significantly lower number of tetanic failures than age-matched control nerves.

In order to study neuromuscular transmission, it is frequently necessary to suppress muscle contractions. In experiments presented in Figures 1 and 2 this was done by using external solutions containing lower calcium and higher magnesium than normal. In such conditions the quantal content of the synaptic transmission is relatively low and thus the e.p.p.s are subthreshold. The obvious question arises of whether the difference between normal and diabetic nerve-muscle preparations could also be observed when the nerve terminal is exposed to a more physiological environment. We therefore repeated the experiments $(n=6)$ in a normal Ringer's solution $([\mathrm{Ca}]=2.0 \mathrm{mM}$, $[\mathrm{Mg}]=1 \mathrm{~mm})$, using $d$-tubocurarine $(0.9 \mu \mathrm{M})$ to prevent muscle contraction. The results were very similar to those in low-calcium Ringer's: in diabetic nerves (duration of diabetes 6 weeks), there were fewer failures upon tetanic stimulation than in normal age-matched rats. [The average number of e.p.p.s in $1 \mathrm{sec}$ during the last $5 \mathrm{sec}$ of a $100 \mathrm{~Hz}, 40 \mathrm{sec}$ nerve stimulation was $98.7 \pm 0.8$ in the diabetic fibers compared with $56.3 \pm 2.4$ in the normal age-matched nerves $(p<0.0005)$.]

\section{Tetanic failures are due to action potential propagation failure}

In many preparations, it is well established that failures in neuromuscular transmission during tetanic stimulation originate mainly from lack of propagation of the action potential into the nerve terminal (Krnjevic and Miledi, 1958; Hatt and Smith, 1976; Spira et al., 1976; Grossman et al., 1979a; Smith, 1980).

We confirmed this finding in the present comparative study by 2 sets of experiments. Table 2 shows that failures in the postsynaptic response in normal and diabetic fibers are not due to the Poisson nature of transmitter release (Del Castillo and Katz, 1954; Boyd and Martin, 1956), and hence they probably originate from lack of activation of the nerve terminals. To test

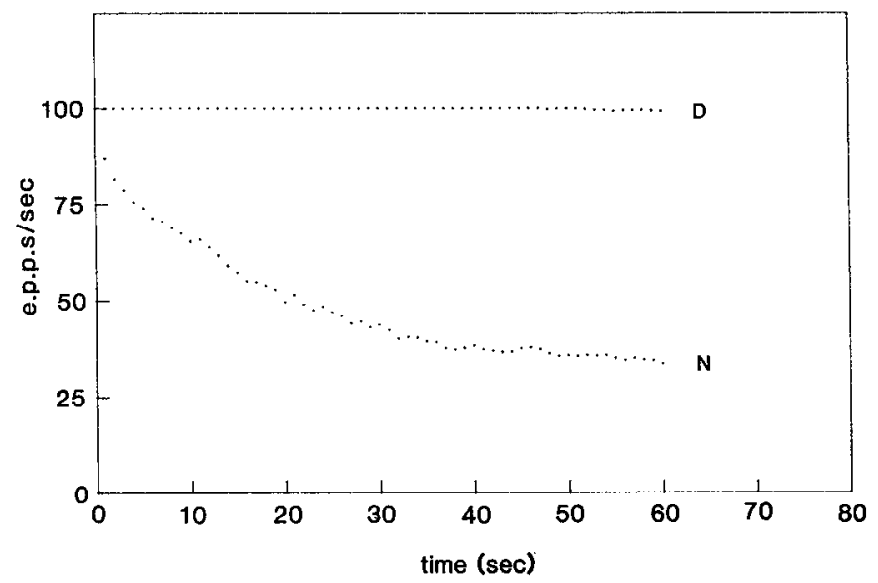

Figure 2. Tetanic failures in diabetic and age-matched control nerve muscle preparations. The average number of successful e.p.p.s in $1 \mathrm{sec}$ (100 minus number of tetanic failures) is plotted as a function of the stimulation duration during a $100 \mathrm{~Hz}, 60 \mathrm{sec}$ nerve stimulation. $D$, Diabetic rats (19 fibers from 5 rats with a diabetes duration of $28-30$ weeks; the mean plasma glucose was $42.4 \pm 0.8 \mathrm{~mm}$ and the mean weight was $192 \pm 14 \mathrm{gm}$ ). $N$, Normal age-matched control rats (21 fibers from 7 rats; the mean plasma glucose was $6.2 \pm 0.4 \mathrm{~mm}$ and the mean weight was $505 \pm 14 \mathrm{gm}$ ). Note the reduction in the number of tetanic failures in the diabetic nerves compared with the normal age-matched control nerves.

this conclusion, we recorded action potentials from the nerve terminals during high-frequency stimulation $(100 \mathrm{~Hz})$ using extracellular recordings. The results of this experiment confirmed that no action potential invaded the nerve terminal when transmission failure occurred (Fig. 3); thus, tetanic failures are caused by a failure of action potential propagation. These results were reproduced in 4 additional normal fibers.

\section{Number of tetanic failures is dependent on age in normal rats}

The number of tetanic failures is decreased in the diabetic motor nerve. We were interested in characterizing the dependence of tetanic failures on the duration of diabetes. However, if the disease was induced at the same age, increased duration of diabetes meant that the animals were older. Therefore, we first had to characterize the dependence of tetanic failures on age in normal rats.

We found that the number of tetanic failures in normal rats is strongly dependent on age. The smallest number of tetanic failures was observed in rats 25 and $36 \mathrm{~d}$ old. At $14 \mathrm{~d}$, the number of tetanic failures was much higher (Fig. $4 a$ ), while in older rats ( $7-36$ weeks), the number of tetanic failures gradually

Table 1. Tetanic failures in normal and diabetic neuromuscular preparations

\begin{tabular}{|c|c|c|c|c|c|}
\hline \multirow[b]{2}{*}{$\begin{array}{l}\text { Diabetes } \\
\text { duration }\end{array}$} & \multicolumn{2}{|l|}{ Diabetes } & \multicolumn{2}{|c|}{ Age-matched controls } & \multirow[b]{2}{*}{$p$} \\
\hline & $\begin{array}{l}\text { E.p.p.s/sec } \\
\pm \text { SEM }\end{array}$ & $\begin{array}{l}\text { Number } \\
\text { of fibers }\end{array}$ & $\begin{array}{l}\text { E.p.p.s/sec } \\
\pm \text { SEM }\end{array}$ & $\begin{array}{l}\text { Number } \\
\text { of fibers }\end{array}$ & \\
\hline $6 \mathrm{~d}$ & $81.0 \pm 6.1$ & 30 & $77.0 \pm 7.1$ & 29 & $<0.2$ \\
\hline $19 \mathrm{~d}$ & $93.8 \pm 3.1$ & 31 & $62.4 \pm 6.4$ & 28 & $<0.0005$ \\
\hline 5-7 weeks & $97.5 \pm 2.0$ & 33 & $49.8 \pm 4.6$ & 41 & $<0.0005$ \\
\hline 28-30 weeks & $100.0 \pm 0.0$ & 19 & $38.8 \pm 3.8$ & 21 & $<0.0005$ \\
\hline
\end{tabular}

The average number of e.p.p.s in a second (mean \pm SEM) during the last $5 \mathrm{sec}$ of a $100 \mathrm{~Hz}, 40 \mathrm{sec}$ nerve stimulation is presented in 4 rat groups with different durations of diabetes and their age-matched control rats. Except for the $6 \mathrm{~d}$ duration of diabetes, in all other groups the average number of e.p.p.s in $1 \mathrm{sec}$ differ significantly between the diabetic and the normal age-matched groups. 


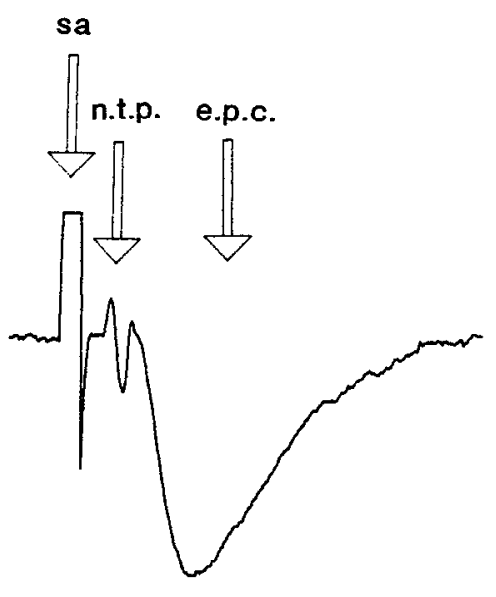

SUCCESSFUL INVASION

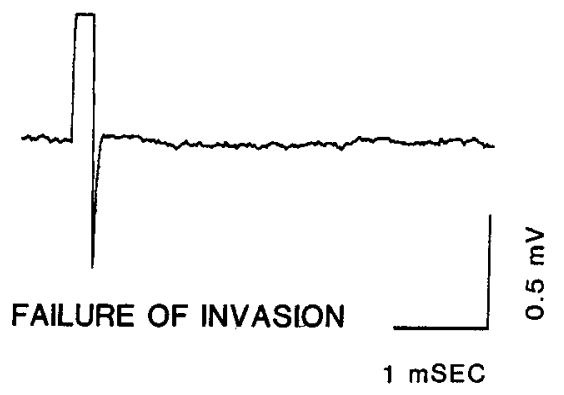

Figure 3. Propagation failure of the action potential into the nerve terminal. Single sweeps recorded extracellularly from a single end plate of an 80-d-old normal rat during a $100 \mathrm{~Hz}, 40 \mathrm{sec}$ nerve stimulation. Upper trace, A "successful" synaptic transmission, in which the stimulation artifact ( $s a$ ), the nerve terminal potential (n.t.p.), and the end plate current (e.p.c.) are all recorded. Lower trace, A tetanic failure, in which only the stimulation artifact is recorded while no e.p.c. nor n.t.p. appear. Thus, during a tetanic failure no action potential invades the nerve terminal.

increased with age (Fig. $4 b$ ). Figure $4 c$ compares the average number of e.p.p.s in $1 \mathrm{sec}$ during the last $5 \mathrm{sec}$ of a $100 \mathrm{~Hz}, 40$ sec nerve stimulation, in normal rats between the ages of 14 and $252 \mathrm{~d}$. This figure clearly demonstrates that the number of tetanic failures is dependent on age in a biphasic manner, with a minimum at 25 and $36 \mathrm{~d}$. The finding that the number of tetanic failures increased with age in rats older than a month is consistent with the finding reported by Smith (1979) that the number of tetanic failures observed in old rats (24-26 months) is greater than that observed in younger rats (12-13 months).

\section{Duration of diabetes and tetanic failures}

Figure 5 compares tetanic failures in rats with 4 different durations of diabetes ( $6 \mathrm{~d}, 19 \mathrm{~d}, 5-7$ weeks, and 28-30 weeks). Figure $5 a$ shows that increasing the duration of diabetes reduces the number of tetanic failures throughout the tetanus, while Figure $5 b$ shows the same phenomenon on the maximal effect.

Figures 2, 6, and 7 and Table 1 compare the number of tetanic failures in diabetic nerves with $6 \mathrm{~d}, 19 \mathrm{~d}, 5-7$ weeks, and 2830 weeks of diabetes to age-matched controls. This comparison was performed because the number of tetanic failures in normal motor nerves depends on age.

After $6 \mathrm{~d}$ of diabetes there was no significant difference between the number of tetanic failures in diabetic nerves and agematched control nerves (Fig. 6a, Table 1). This finding indicates that a certain minimal duration of diabetes is necessary to produce the resistance to high-frequency stimulation. This result has also a methodological value. It rules out the possibility that the relative resistance of the diabetic nerve to high-frequency stimulation is caused by a direct and immediate effect of STZ, a dircet and immediate effect of hypcrglycemia, or an osmotic and glucose concentration difference during the experiment. (The serum glucose concentration was $30-40 \mathrm{~mm}$ in diabetic rats, 6$8 \mathrm{~mm}$ in normal rats, and $11 \mathrm{~mm}$ in the bath solution during the electrophysiological experiments.)

The shortest duration of diabetes in which we found a significant reduction in the number of tetanic failures in diabetic nerves was $19 \mathrm{~d}$ (Fig. 7, Table 1) (no data points were obtained between 6 and $19 \mathrm{~d}$ ). The reduction in the number of tetanic failures in diabetic nerves persisted after 5-7 and 28-30 weeks of diabetes (Figs. $6 b$ and 2, respectively). Furthermore, the difference between the number of tetanic failures in diabetic motor nerves and normal age-matched nerves increased with the duration of diabetes. This phenomenon is caused by a combined effect of a reduction in the number of tetanic failures with increasing duration of diabetes and an increase in the number of tetanic failures with age in normal nerves.

\section{Insulin and tetanic failures}

It was of interest to examine whether controlling diabetes by daily insulin injections could prevent the reduction in the number of tetanic failures in the diabetic nerve. We therefore compared tetanic failures in 3 rat groups: (1) diabetic rats (duration

Table 2. Statistical properties of transmission failure

Normal

\begin{tabular}{|c|c|c|c|c|c|c|c|}
\hline \multicolumn{2}{|c|}{$\begin{array}{l}\text { Percent failures } \\
(\text { mean } \pm \text { SEM })\end{array}$} & \multirow{2}{*}{\multicolumn{2}{|c|}{$\begin{array}{l}\text { Num- } \\
\text { ber of } \\
\text { fibers } p\end{array}$}} & \multicolumn{2}{|c|}{$\begin{array}{l}\text { Percent failures } \\
(\text { mean } \pm \text { SEM) }\end{array}$} & \multirow{2}{*}{$\begin{array}{l}\text { Num- } \\
\text { ber of } \\
\text { fibers }\end{array}$} & \multirow{2}{*}{$p$} \\
\hline Observed & Expected & & & Observed & Expected & & \\
\hline $56.8 \pm 4.1$ & $19.3 \pm 3.6$ & 12 & $<0.0005$ & $46 \pm 5.5$ & $10.4 \pm 2.6$ & 5 & $<0.0005$ \\
\hline
\end{tabular}

The average percentage of synaptic failures observed (mean \pm SEM) during the last $10 \mathrm{sec}$ of a $100 \mathrm{~Hz}, 40 \mathrm{sec}$ nerve stimulation are compared with the percentage of synaptic failures expected (mean \pm SEM) from the quantal content (mean e.p.p. amplitude/mean miniature e.p.p. amplitude) during the same period of time assuming the failures are Poissonian in nature in both diabetic (duration of diabetes 5-7 weeks) and age-matched control fibers. The fibers chosen were those which showed a large number of tetanic failures during the high-frequency stimulation. In both groups, the observed synaptic failures differ significantly from the expected. We conclude that tetanic failures in both diabetic and control preparations are not due to the stochastic nature of transmitter release. 


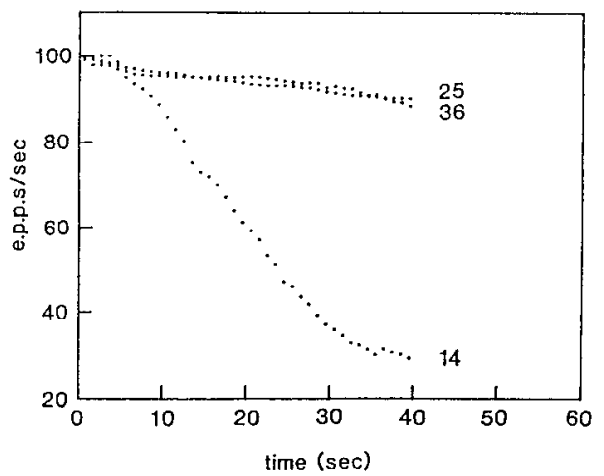

c

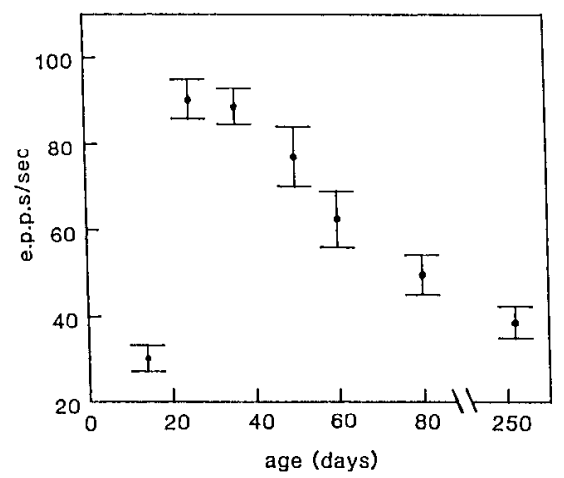

b

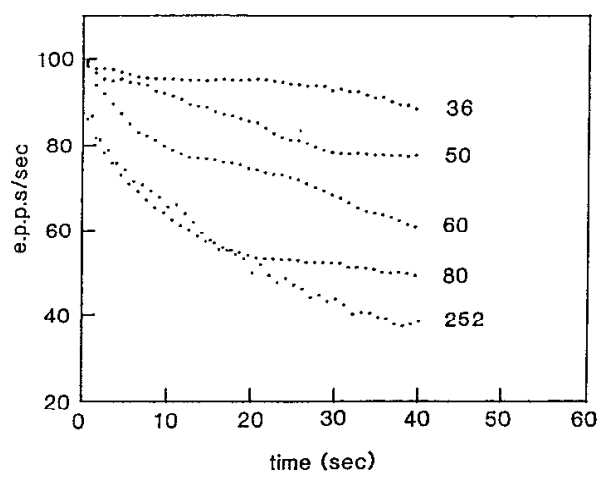

Figure 4. The number of tetanic failures is dependent on age in normal rats. $a$ and $b$, The average number of successful e.p.p.s in $1 \mathrm{sec}$ is plotted as a function of the stimulation duration during a $100 \mathrm{~Hz}, 40 \mathrm{sec}$ nerve stimulation in nerves taken from normal rats with different ages: $14 \mathrm{~d}$ (24 fibers), 25 $\mathrm{d}$ (30 fibers), $36 \mathrm{~d}$ (36 fibers), 7 weeks (50 d, 29 fibers), 9 weeks (60 d, 28 fibers), 11-13 weeks ( $80 \mathrm{~d}, 41$ fibers), and 35-37 weeks (252 d, 19 fibers). $c$, The average number of successful e.p.p.s in $1 \mathrm{sec}( \pm$ SEM) during the last $5 \mathrm{sec}$ of a $100 \mathrm{~Hz}, 40 \mathrm{sec}$ nerve stimulation is plotted as a function of age in normal nerves. The statistical analysis of the results presented shows that the differences are significant $(p<0.005)$ for the comparison of 14 and $25 \mathrm{~d}, 36 \mathrm{~d}$ and 9 weeks, 7 and $11-13$ weeks, 9 and 3537 weeks. $p=0.05$ for the comparison of 9 and 11-13 weeks. Note the biphasic dependence of the number of tetanic failures on age in normal nerves. of diabetes $19 \mathrm{~d}) ;(2)$ similar diabetic rats treated with 2 daily insulin injections of an average daily dose of 6-7 U/d (glucose levels were kept below 6-7 $\mathrm{mm}$ ); and (3) age-matched control rats.

The results of these experiments are plotted in Figure 7. The conclusion is clear: the number of tetanic failures in the insulintreated diabetic nerve muscle preparation was much higher than that in the untreated preparations and was not significantly different from the number of failures in normal age-matched preparations. Thus, insulin treatment does prevent the development of the diabetic nerve relative resistance to high-frequency stimulation.
We were interested in finding out whether a short exposure of diabetic nerves to insulin in vitro can also reverse the reduction in the number of tetanic failures. To answer this question we examined tetanic failures in diabetic fibers (duration of diabetes $5-7$ weeks) incubated with insulin $(60 \mathrm{mU} / \mathrm{ml})$ in vitro for several hours.

The mean number of e.p.p.s/sec during the last $5 \mathrm{sec}$ of a 40 sec, $100 \mathrm{~Hz}$ nerve stimulation was $100.0 \pm 0.0$ in the diabetic fibers incubated with insulin (19 fibers from 3 rats, 6 of which were incubated with insulin for $1 \mathrm{hr}$ and the remaining 13 fibers incubated $8 \mathrm{hr}$ ).

We conclude that controlling diabetes with insulin can pre- a

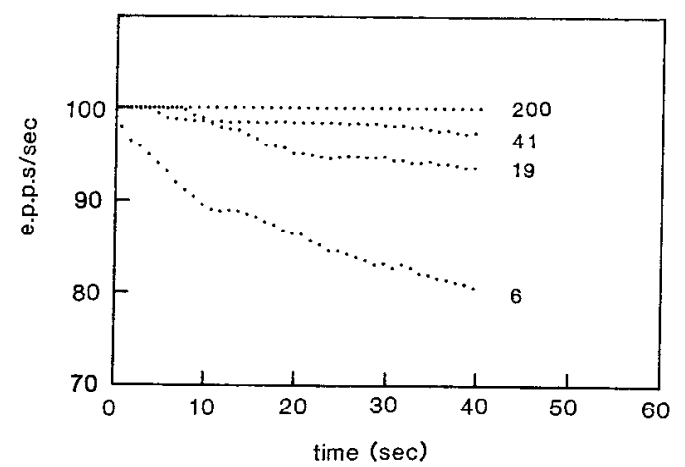

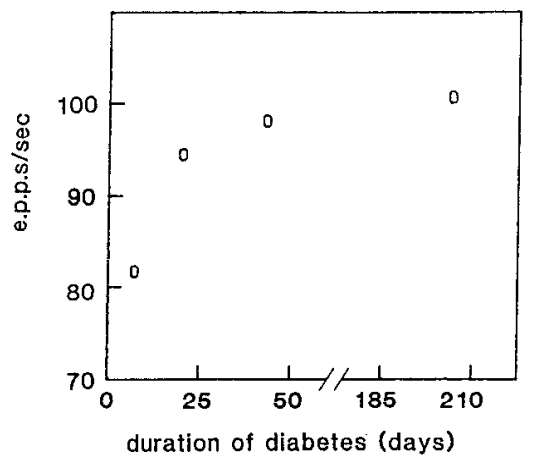

Figure 5. Tetanic failures decrease in number with increasing diabetes duration. $a$, The average number of successful e.p.p.s in a second is plotted as a function of the stimulation duration during a $100 \mathrm{~Hz}, 40 \mathrm{sec}$ nerve stimulation for different durations of diabetes: $6 \mathrm{~d}, 19 \mathrm{~d}, 5-7$ weeks $(41 \mathrm{~d})$, and 28-30 weeks $(200 \mathrm{~d}) . b$, The average number of successful e.p.p.s in $1 \mathrm{sec}$ during the last $5 \mathrm{sec}$ of a $100 \mathrm{~Hz}, 40 \mathrm{sec}$ nerve stimulation is plotted as a function of the diabetes duration in the different diabetic groups. Note the reduction in the number of tetanic failures with increasing duration of diabetes. 
a

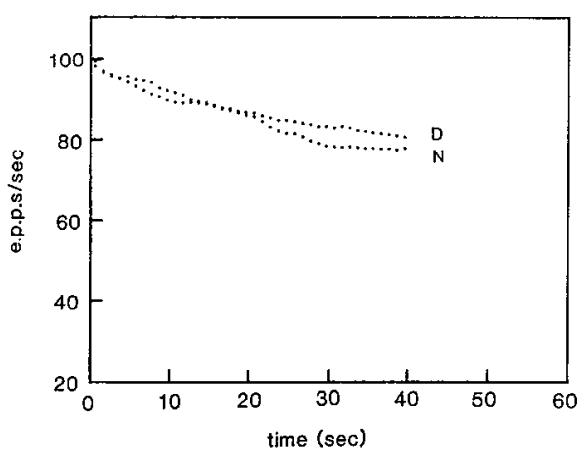

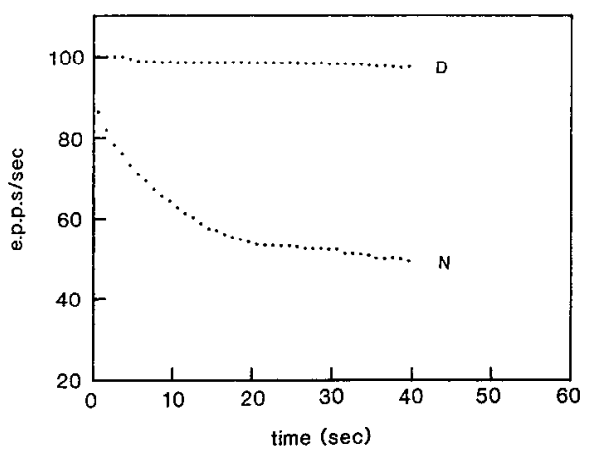

Figure 6. Comparison of tetanic failures in diabetic and normal age-matched control rats with different durations of diabetes. The average number of successful e.p.p.s in a second is plotted as a function of the stimulation duration during a $100 \mathrm{~Hz}, 40 \mathrm{sec}$ nerve stimulation. $a$, Diabetic rats with $6 \mathrm{~d}$ of diabetes ( 30 fibers from 5 rats; mean plasma glucose, $30.5 \pm 1.1 \mathrm{~mm}$; mean weight, $210 \pm 3.5$ gm) and age-matched control rats (29 fibers from 5 rats; mean plasma glucose, $6.5 \pm 0.4 \mathrm{~mm}$; mean weight, $214 \pm 5.7 \mathrm{gm}$ ). $b$, Diabetic rats with $5-7$ weeks of diabetes (33 fibers from 6 rats; mean plasma glucose, $32.0 \pm 1.3 \mathrm{~mm}$; mean weight, $182 \pm 15 \mathrm{gm}$ ) and age-matched control rats (41 fibers from 8 rats; mean plasma glucose, $6.1 \pm 0.3 \mathrm{~mm}$; mean weight, $346 \pm 10.9 \mathrm{gm})$. In both groups, $\bar{D}$ and $N$ stand for diabetes and normal age-matched controls, respectively.

vent the development of the diabetic nerve relative resistance to high-frequency stimulation, while incubating the diabetic nerve with insulin in vitro for several hours does not produce the same effect.

\section{Terminal intramuscular branches are responsible for the reduction in the number of tetanic failures in diabetic motor nerves}

Diabetic nerves show a reduction in the number of tetanic failures compared with that of normal age-matched control nerves. Earlier we had shown that tetanic failures are caused by a propagation failure of the action potential into the presynaptic nerve terminal.

To localize the axonal site responsible for the diabetic nerve's relative resistance to high-frequency stimulation, we recorded

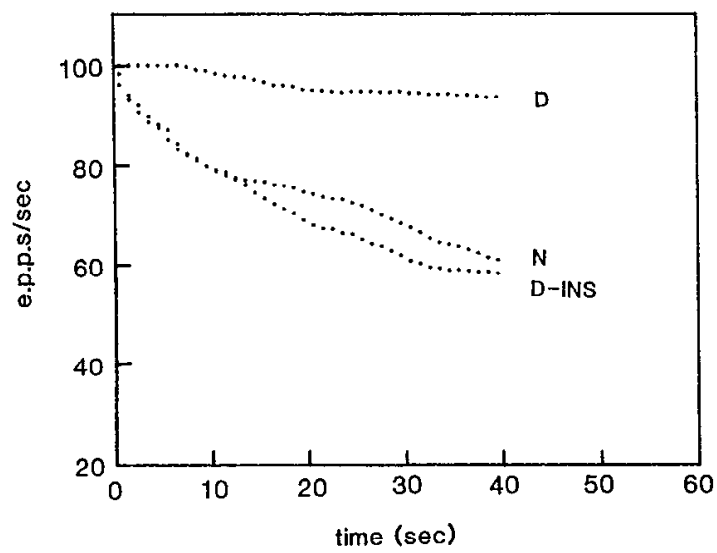

Figure 7. Effect of daily insulin injections on the number of tetanic failures in diabetic rats. The average number of successful e.p.p.s in 1 sec is plotted as a function of the stimulation duration during a $100 \mathrm{~Hz}$, $40 \mathrm{sec}$ nerve stimulation, $D$, Diabetic rats ( 31 fibers from 5 rats with a diabetes duration of $19 \mathrm{~d}$; mean plasma glucose, $35.7 \pm 0.7 \mathrm{~mm}$; mean weight, $186 \pm 8.7 \mathrm{gm})$. $D-I N S$, Similar diabetic rats treated with 2 daily insulin injections ( 25 fibers from 5 rats; mean plasma glucose, $6.5 \pm$ $0.3 \mathrm{~mm}$; mean weight, $225 \pm 9 \mathrm{gm}$ ). $N$, Normal age-matched control rats (28 fibers from 5 rats; mean plasma glucose, $6.1 \pm 0.2 \mathrm{~mm}$; mean weight, $236 \pm 8.7 \mathrm{gm})$. Controlling diabetes by insulin injections prevented the development of a relative resistance to high frequency in diabetic nerves. extracellular compound action potentials (c.a.p.s) from the sciatic nerve and the proximal intramuscular branches innervating the soleus muscle. The results of these experiments show that the c.a.p. amplitude is reduced during high-frequency stimulation from both sciatic nerves and proximal intramuscular branches (Fig. 8, $a, b$ and $c, d$, respectively). The reduction in the c.a.p. amplitude may result from several mechanisms: a reduction in axonal action potential amplitude, a block of axonal action potential conduction in a portion of the axons, or failure of action potential initiation at the site of nerve stimulation. Whatever the reason for the reduction in c.a.p. amplitude during high-frequency stimulation, our results show that this reduction in both sciatic nerve and proximal intramuscular branches is not significantly different in normal and diabetic nerves (Fig. 8). Thus, we conclude that most, if not all, tetanic failures seen in normal motor nerves are caused by an intermittent block of the conduction of action potentials along the distal intramuscular branches. Furthermore, the relative resistance of the diabetic nerve to high-frequency stimulation is caused by a reduction in the intermittent block of the action potentials, along the small intramuscular branches of the motor nerve. This results in a larger number of action potentials invading the diabetic nerve terminal to generate an e.p.p.

\section{Ionic mechanisms responsible for the smaller number of failures in the diabetic nerve}

Several ionic mechanisms may explain the diabetic nerve relative resistance to high-frequency stimulation. We examined 2 such mechanisms, which involve the sodium-potassium pump and extracellular potassium.

The sodium-potassium pump was shown to play 2 opposing roles in tetanic failure formation in different preparations. In some preparations, such as sensory neurons of the leech and rat demyelinated ventral spinal roots, inhibiting the sodium-potassium pump by ouabain or lithium decreases the number of tetanic failures (Van Essen, 1973; Bostock and Grafe, 1985); in other preparations, the opposite occurs (Grossman et al., 1979b; Kocsis et al., 1983).

The activity of the sodium-potassium pump is decreased in the diabetic nerve (Das et al., 1976; Greene et al., 1985, 1987; Winegrad, 1987). If tetanic failures seen in the motor nerve are 
a

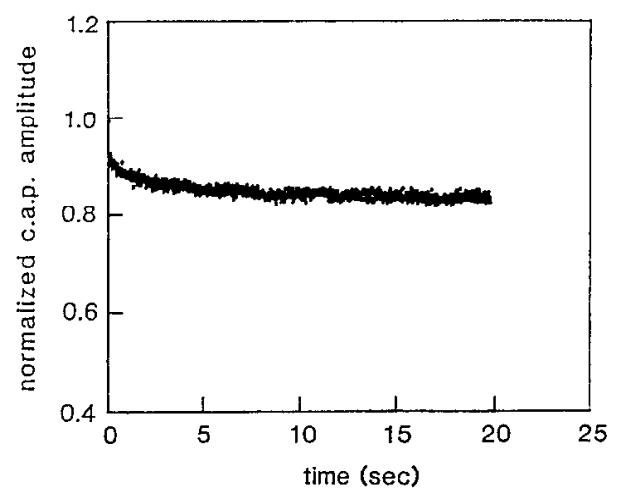

b

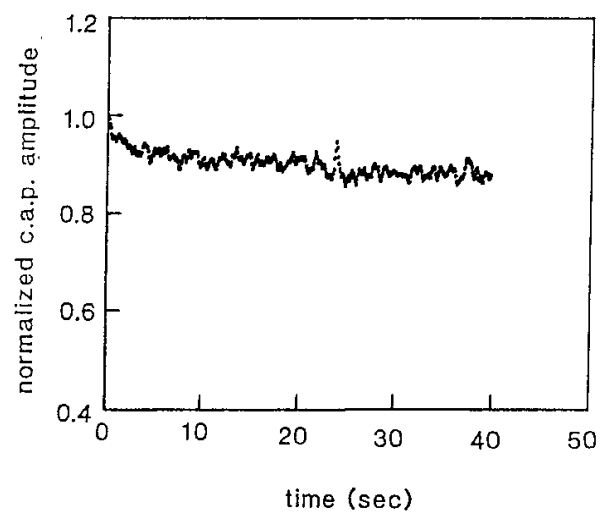

diabetes

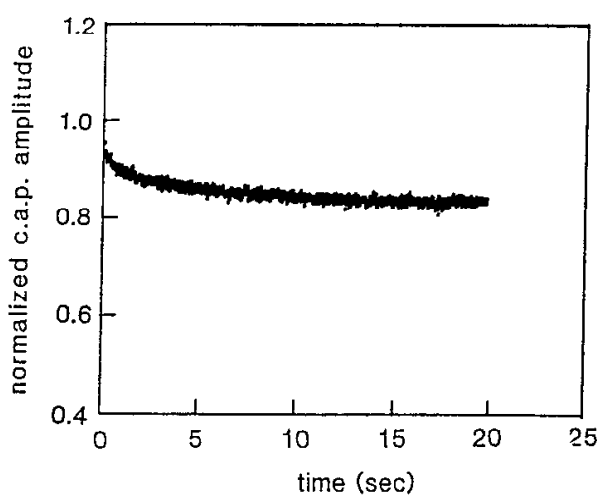

diabetes

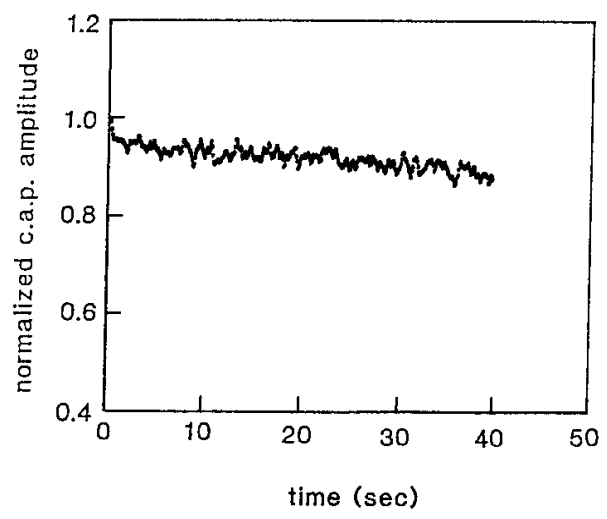

Figure 8. The compound action potential amplitude during highfrequency stimulation in diabetic and age-matched controls. The average normalized compound action potential amplitudes (c.a.p.) are plotted as a funclion of the stimulation duration during a $100 \mathrm{~Hz}$ nerve stimulation. $a$, c.a.p.s recorded from sciatic nerves of diabetic (11 nerves from 6 rats, with a diabetes duration of 6-7 weeks; mean plasma glucose, $39.4 \pm 0.7 \mathrm{~mm}$; mean weight, $220.0 \pm 7.2 \mathrm{gm})$ and age-matched controls $(11$ nerves from 6 rats; mean plasma glucose, $6.0 \pm 0.2 \mathrm{~mm}$; mean weight $374 \pm 6.8 \mathrm{gm})$. The average normalized c.a.p. amplitude from the last 50 c.a.p.s of the $100 \mathrm{~Hz}, 20 \mathrm{sec}$ nerve stimulation was $0.834 \pm 0.003$ and $0.834 \pm 0.004$ in diabetic and age-matched controls respectively $(p>0.5) . b$, c.a.p.s recorded from the proximal intramuscular branches innervating the soleus muscle of diabetic ( 8 nerves from 6 rats with a diabetes duration of 5-7 weeks; mean plasma glucose, $34.7 \pm 1.1 \mathrm{~mm}$; mean weight, $196 \pm 4.1 \mathrm{gm}$ ) and age-matched controls $(7$ nerves from 6 rats; mean plasma glucose, $6.8+0.4 \mathrm{~mm}$; mean weight, $385 \pm 10.4 \mathrm{gm})$. The average normalized c.a.p. amplitude from the last 50 c.a.p.s of the $100 \mathrm{~Hz}, 40 \mathrm{sec}$ nerve stimulation was $0.875 \pm 0.005$ and $0.878 \pm 0.005$ in diabetic and agematched controls respectively $(p>0.3)$. Note that the reduction in the c.a.p. amplitudes in both sciatic nerve and proximal intramuscular branches were not significantly different between diabetic and age-matched controls. In $a$ and $b$, each second and sixth c.a.p.s were randomly analyzed, respectively.

caused by an activation of the sodium-potassium pump and hyperpolarization of the membrane resting potential, then the reduction in the activity of the pump, described in the diabetic nerve, might explain the differences in tetanic failures between normal and diabetic nerves.

To test the validity of this possibility we examined the effect of ouabain $(0.2 \mathrm{~mm})$ on tetanic failures in normal nerves (exposed to the inhibitor $10 \mathrm{~min}$ prior to nerve stimulation). We found that ouabain markedly increased the number of failures in normal nerves (Fig. 9), which is inconsistent with the proposed mechanism.

The relative resistance to high-frequency stimulation could be explained by a second mechanism involving a change in the sodium-potassium pump activity: since the axonal caliber of the diabetic nerve is decreased (Jakobsen, 1976, 1978; Brown et al., 1980; Robertson and Sima, 1980), the internal volume of the diabetic nerve decreases more than the surface area of its axolemma. This can lead to a greater amount of sodium accumulating in the axoplasm, which in turn may activate the sodium-potassium pump, decrease the potassium concentration in the extracellular space surrounding the axon, and reduce the number of tetanic failures in the diabetic nerve (Grossman et al., 1979b). To test this hypothesis we examined the effect of ouabain ( $0.2 \mathrm{~mm} 10 \mathrm{~min}$ prior to nerve stimulation) on the

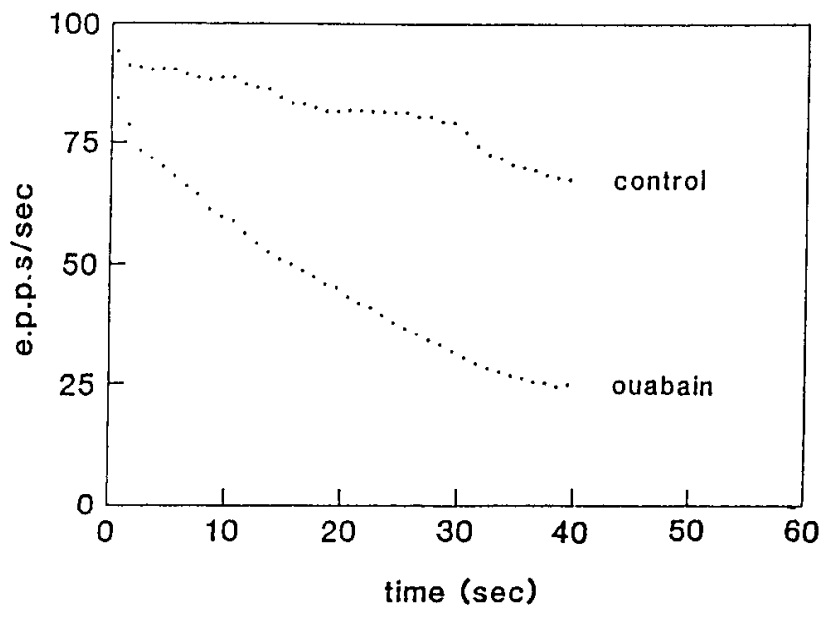

Figure 9. Ouabain increases the number of tetanic failures in normal nerves. The average number of successful e.p.p.s in a second is plotted as a function of the stimulation duration during a $100 \mathrm{~Hz}, 40 \mathrm{sec}$ nerve stimulation. Ten normal nerve fibers were examined, both before (control) and after a $10 \mathrm{~min}$ exposure to $0.2 \mathrm{~mm}$ ouabain. The average number of e.p.p.s in a second during the last $5 \mathrm{sec}$ of the $100 \mathrm{~Hz}, 40$ sec nerve stimulation was $68.3 \pm 8.0$ before and $25.1 \pm 5.4$ after the application of ouabain $(p<0.0001)$. It is evident from these results that ouabain increases the number of tetanic failures in normal nerves. 

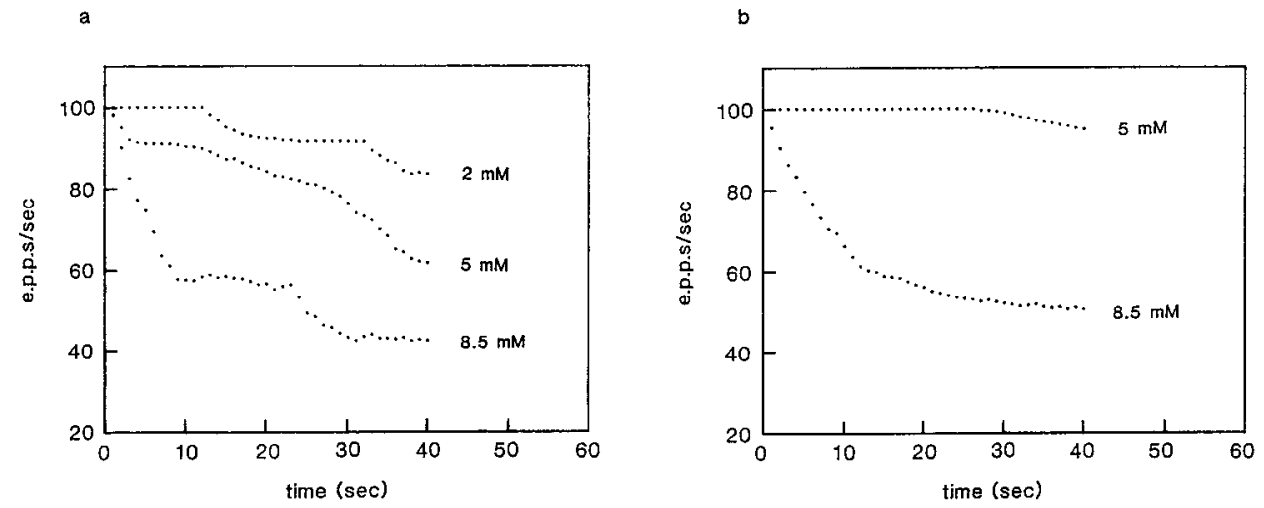

Figure 10. Tetanic failures are dependent on extracellular potassium concentrations. The average number of successful e.p.p.s in 1 sec are plotted as a function of the stimulation duration during a $100 \mathrm{~Hz}, 40 \mathrm{sec}$ nerve stimulation at different extracellular potassium concentrations (2, 5 , and $8.5 \mathrm{~mm}$ ). a, Normal nerves (6 fibers from 5 rats), each of which was exposed to the 3 different potassium concentrations. The average numbers of e.p.p.s in $1 \mathrm{sec}$ during the last $5 \mathrm{sec}$ of the $100 \mathrm{~Hz}, 40 \mathrm{sec}$ nerve stimulation were $25.0 \pm 0.8,59.6 \pm 0.2$, and $80.6 \pm 2.3$ for $8.5,5$, and $2 \mathrm{~mm}$ extracellular potassium, respectively $(p<0.0001$ for any comparison). $b$, Diabetic nerves (16 fibers from 3 rats with a diabetes duration of 30 d; mean plasma glucose concentration, $33.2 \pm 1.2 \mathrm{~mm}$; mean weight, $181 \pm 7 \mathrm{gm}$ ). The average number of e.p.p.s in $1 \mathrm{sec}$ during the last $5 \mathrm{sec}$ of a $100 \mathrm{~Hz}, 40 \mathrm{sec}$ nerve stimulation was $96.5 \pm 2.8$ and $55.8 \pm 7.4$ for 5 and $8.5 \mathrm{~mm}$ extracellular potassium, respectively $(p<0.0005)$. Note that raising potassium concentration increases the number of tetanic failures in both diabetic and normal fibers; furthermore, when the extracellular potassium was $8.5 \mathrm{~mm}$, the number of tetanic failures in diabetic nerves was in the range of the number of tetanic failures in normal nerves.

number of tetanic failures in diabetic nerves. If the hypothesis were valid, we expected that with the addition of ouabain the number of tetanic failures would be similar in diabetic and normal nerves. The results of these experiments showed that ouabain increased the number of tetanic failures in the diabetic nerve, but, even after the addition of ouabain, the number of tetanic failures in the diabetic nerve was much smaller than that in normal nerves with and without ouabain. The mean time to the appearance of tetanic failure in diabetic nerves was 113.2

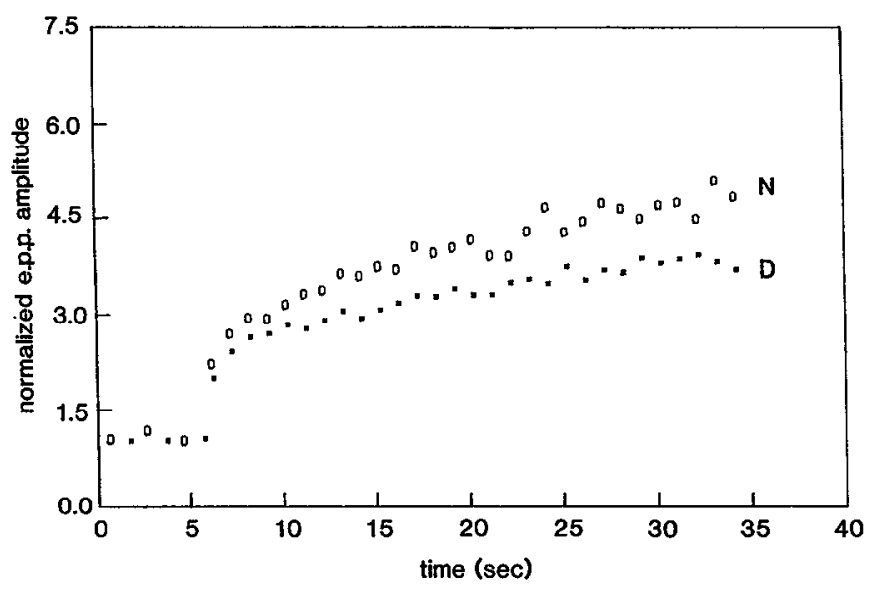

Figure 11. Tetanic potentiation in normal and diabetic nerve muscle preparations. The normalized average e.p.p. amplitude is plotted as a function of the stimulation duration during a $50 \mathrm{~Hz}, 30 \mathrm{sec}$ nerve stimulation in diabetic rats $(D, 26$ fibers from 5 rats with a diabetes duration of 5-7 weeks; mean plasma glucose, $36.0 \pm 1.9 \mathrm{~mm}$; mean weight, 173 $\pm 10 \mathrm{gm})$ and age-matched control rats $(N, 19$ fibers from 7 rats; mean plasma glucose, $6.7 \pm 0.3 \mathrm{~mm}$; mean weight, $361 \pm 11 \mathrm{gm}$ ). In the first $6 \mathrm{sec}$, the stimulation rate was $0.5 \mathrm{~Hz}$. During the $50 \mathrm{~Hz}$ stimulation, each point represents the average of 50 e.p.p.s. The normalized average e.p.p. amplitude in the last second of the $50 \mathrm{~Hz}, 30 \mathrm{sec}$ nerve stimulation was $3.74 \pm 0.08$ and $4.76 \pm 0.11$ in the diabetic nerves and normal nerves, respectively $(p<0.0005)$. In all experiments of this kind, the bath solution contained $0.275 \mathrm{~mm} \mathrm{Ca}$ and $1.5 \mathrm{~mm} \mathrm{Mg}$. Note that tetanic potentiation in the diabetic group is reduced compared with that of the normal age-matched group. \pm 9.1 and $56.0 \pm 7.3 \mathrm{sec}$ before and after the addition of ouabain, respectively. The average number of e.p.p.s/sec during the $5 \mathrm{sec}$ between 35 and $40 \mathrm{sec}$ of the $100 \mathrm{~Hz}$ nerve stimulation was $100 \pm 0.0$ and $97.7 \pm 2.1$ before and after the addition of ouabain, respectively. Thus, we conclude that the hypothesis tested is incorrect.

The last plausible ionic mechanism we examined concerns cxtraccllular potassium accumulation.

During high-frequency stimulation, potassium accumulates in the extracellular fluid surrounding the axons, and its concentration temporarily rises by several millimolars, usually 3-5 (Frankenhaeuser and Hodgkin, 1956; Spira et al., 1976; Nicoll, 1979; Somjen, 1979; Smith, 1983). In most preparations examined, elevation of the bath potassium increases the number of tetanic failures (Spira et al., 1976; Grossman et al., 1979b; Smith, 1979, 1980; Kocsis et al., 1983). In some preparations, the accumulation of potassium may play a major role in the formation of tetanic failures (Grossman et al., 1979b; Kocsis et al., 1983). In others, the role of potassium accumulation in the formation of tetanic failures is less clear (Grossman and Gutnick, 1981; Smith, 1983).

The smaller number of tetanic failures in the diabetic nerve might result from a reduction in potassium accumulating in the periaxonal extracellular space surrounding the diabetic axon during high-frequency stimulation.

Consistent with this hypothesis, we found that the number of tetanic failures in normal nerves is strongly dependent on the bath potassium concentration (Fig. 10a): raising the bath potassium from 5 to $8.5 \mathrm{~mm}$ caused a significant increase in the number of tetanic failures, while lowering the bath potassium from 5 to $2 \mathrm{~mm}$ reduced the number of tetanic failures. Raising the bath potassium concentration from 5 to $8.5 \mathrm{~mm}$ also significantly increased the number of tetanic failures in diabetic nerves (Fig. 10b). Furthermore, the number of tetanic failures in diabetic nerves in these conditions were in the range seen in normal nerves. These findings are thus consistent with the hypothesis that the reduction in the number of tetanic failures in the diabetic nerve is caused by less potassium accumulating in the periaxonal space surrounding the diabetic nerve during high- 
frequency stimulation. We stress that this hypothesis is not proven by the experimental findings and that other mechanisms might also explain the reduction in the number of tetanic failures in the diabetic nerve.

\section{Tetanic potentiation in the normal and diabetic neuromuscular junction}

During high-frequency nerve stimulation the e.p.p. amplitude increases. This phenomenon is caused by the larger number of quanta released from the nerve terminal (Magleby and Zengel, 1975; Lev-Tov and Rahamimoff, 1980; Erulkar, 1983; Nussinovitch and Rahamimoff, 1988). Our initial aim was to examine whether there is a difference in tetanic potentiation between normal and diabetic rats. This was tested on neuromuscular preparations bathed in low-calcium, high-magncsium solutions, in which the quantal content is low.

Our expectations were that diabetic nerves might have a larger tetanic potentiation since the diabetic nerve showed a relative resistance to high-frequency stimulation. To avoid the ambiguity of unequal number of stimuli, we stimulated the nerves with a frequency and a duration that do not produce a significant number of failures. Figure 11 presents the tetanic potentiation in normal and diabetic nerves (duration of diabetes, 5-7 weeks) during a $50 \mathrm{~Hz}, 30 \mathrm{sec}$ nerve stimulation. The tetanic potentiation in diabetic nerves was significantly smaller than that in normal age-matched nerves.

\section{Discussion}

This study compared neuromuscular transmission during highfrequency stimulation in normal and diabetic rats. There were 2 main findings: tetanic potentiation was significantly reduccd at the diabetic neuromuscular junction, and the diabetic nerve muscle preparation was more resistant to high-frequency stimulation than were age-matched controls. The stimulation rates used in this study $(50-100 \mathrm{~Hz})$ are in the upper range of action potential frequency recorded in vivo from the rat soleus muscle (Henning and Lomo, 1985).

We chose to examine neuromuscular transmission during highfrequency stimulation for 2 reasons: high-frequency stimulation is a physiological modulator of synaptic transmission (for review, see Erulkar, 1983), and we believed that a stressing condition such as high-frequency stimulation might exaggerate changes in the diabetic nerve, and thus enable us to examine minor changes barely expressed in normal conditions.

The appearance of synaptic transmission failure during highfrequency stimulation is well documented in several vertebrate and invertebrate preparations, including the neuromuscular junction of the rat (Krnjevic and Miledi, 1958, 1959; Spira et al., 1976; Grossman et al., 1979a, b; Smith, 1979, 1980; Kocsis et al., 1983). In this study it was shown that, similar to other preparations, tetanic failure in the rat neuromuscular junction is caused by a propagation failure of action potentials into the nerve terminals and not by the Poissonian nature of transmitter release.

The diabetic nerve is relatively resistant to high-frequency stimulation and the number of tetanic failures in it is decreased compared with their number in normal age-matched nerves. This finding was unexpected. In diabetic nerves a reduction of fast sodium currents was observed, caused by a reduction of the driving force of sodium and a larger fraction of sodium channels being in the inactivated state (Brismar and Sima, 1981; Brismar, 1983; Brismar et al., 1987). This reduction of fast sodium cur- rents would be expected to cause a decrease in action potential amplitude and, as a result, to increase the number of tetanic failures.

Using extracellular recordings of compound action potentials from several locations along the nerve, we found that the site responsible for the reduction in the number of tetanic failures in the diabetic nerve is located at the distal intramuscular branches between the end plate and the proximal intramuscular branches.

The diabetic nerve's relative resistance to high-frequency stimulation has certain similarities with another paradoxical phenomenon reported in diabetic nerves of both patients and animal models: a relative resistance to ischemia (Steiness, 1959; Horowitz and Ginsberg-Fellner, 1979; Nielsen, 1982; Low, 1987). However, the 2 phenomena differ in their time course. The relative resistance to ischemia appears only $2 \mathrm{hr}$ after the induction of diabetes or hyperglycemia in normal rats (Shirabe et al., 1988), whereas the relative resistance to high-frequency. stimulation develops within weeks after the induction of diabetes.

In this work we examined several possible mechanisms that may contribute to the diabetic nerve's relative resistance to highfrequency stimulation. Two of them regarding the possible role of the pump do not seem very likely, as discussed in Results. The third possibility, namely, less $\mathrm{K}^{+}$accumulation outside diabetic than outside normal nerves, is consistent with the experimental data.

We found that the number of tetanic failures increases with the extracellular potassium concentration in both diabetic and normal nerves. Furthermore, we found that raising the extracellular potassium concentration by $3.5 \mathrm{~mm}$ increased the number of tetanic failures in the diabetic nerve and brought it into the range seen in age-matched control nerves. The value of 3.5 $\mathrm{mm}$ is within the range of the $3-5 \mathrm{mM}[\mathrm{K}]_{0}$ increase found during high-frequency stimulation in other preparations (Frankenhaeuser and Hodgkin, 1956; Spira et al., 1976; Nicoll, 1979; Somjen, 1979). These findings are consistent with the hypothesis that the relative resistance of the diabetic nerve to high-frequency stimulation is caused by a reduction in $\mathrm{K}^{+}$accumulation in the extracellular space surrounding the axon. The effect of elevating extracellular $\mathrm{K}^{+}$concentration in the bath solution can be mediated by a direct effect on the membrane potential.

Several mechanisms might reduce potassium accumulation in the diabetic periaxonal extracellular space during high-frequency stimulation: increased endoneural volume in diabetic nerves (Jakobsen, 1978; Nielsen, 1982), lower intracellular potassium concentrations in diabetic axons (Sjogren et al., 1986), or more permeable diffusion barriers, which normally limit the diffusion of potassium out of the perineural space (Senevirante and Peiris, 1970).

Other possible mechanisms unrelated to $\mathrm{K}^{+}$accumulation that might explain the diabetic nerve relative resistance to highfrequency stimulation include the following:

1. Certain anatomical changes such as reduction in the number of bifurcating sites along the axon due to intramuscular branch degeneration or changes in the geometric ratio (Rall, 1962; Parnas and Segev, 1979) could decrease the number of tetanic failures in diabetic nerves.

2. The diabetic nerve has increased energy stores (Greene and Winegrad, 1981; Low et al., 1985; Low, 1987). There is a possibility that the diabetic nerve's ability to better cope with stressing conditions, such as ischemia or high-frequency stimulation, 
is the result of this phenomenon (Low et al., 1985; Low, 1987). Whatever the mechanism of this diabetic resistance to highfrequency stimulation, it is clear that it partially ameliorates the basic impairment in neuromuscular transmission present in diabetes.

\section{References}

Bostock, H., and P. Grafe (1985) Activity dependent excitability changes in normal and demyelinated rat spinal root axons. J. Physiol. (Lond.) 365: 239-257.

Boyd, I. A., and A. R. Martin (1956) The end plate potential in mammalian muscle. J. Physiol. (Lond.) 132: 74-91.

Brismar, T. (1983) Neuropathy-functional abnormalities in the BB Wistar rat. Metabolism 32: 112-117.

Brismar, T., and A. A. F. Sima (1981) Changes in nodal function in nerve fibers of spontaneously diabetic BB Wistar rat: Potential clamp analysis. Acta Physiol. Scand. 113: 499-506.

Brismar, T., A. A. F. Sima, and D. A. Greene (1987) Reversible and irreversible nodal dysfunction in diabetic neuropathy. Ann. Neurol. 21: 504-507.

Brown, M. J., and A. K. Asbury (1984) Diabetic neuropathy. Ann. Neurol. 15: 2-12.

Brown, M. J., A. J. Summer, D. A. Greene, S. A. Diamond, and A. K. Asbury (1980) Distal neuropathy in experimental diabetes mellitus. Ann. Neurol. 8: 168-178.

Byrne, P. J., and P. S. Schein (1981) Streptozotocin. In Nitrosurea: Current Status and New Developments, A. W. Prestanko, L. H. Baker, S. T. Crooke, S. K. Carter, and P. S. Schein, eds., pp. 367-377, Academic, New York.

Clements, R. S. (1979) Diabetic neuropathy-New concepts of its etiology. Diabetes 28: 604-611.

Constantini, S., Y. Schiller, A. M. Cohen, and R. Rahamimoff (1987) Pathophysiology of the neuromuscular junction in diabetic rats. Isr. J. Med. Sci. 23: 101-105.

Das, P. K., E. M. Bray, A. J. Aguayo, and M. S. Raminsky (1976) Diminished ouabain sensitive $\mathrm{Na} / \mathrm{K}$ ATPase activity in sciatic nerves of rats with streptozotocin induced diabetes. Exp. Neurol. 53: 285288.

Del Castillo, J., and B. Katz (1954) Quantal components of the end plate potential. J. Physiol. (Lond.) 124: 560-573.

Dyck, P. J., J. L. Karnes, J. Daube, P. O'Brian, and F. J. Service (1985) Clinical and neuropathological criteria for diagnosis and staging of diabetic polyneuropathy. Brain 108: 861-880.

Dyck, P. J., J. L. Karnes, P. C. O'Brian, and E. J. Swanson (1986) Neuropathy symptom profile in health, motor neuron disease, diabetic neuropathy and amyloidosis. Neurology 36: 1300-1308.

Erulkar, S. D. (1983) The modulation of neurotransmitter release at synaptic junctions. Rev. Physiol. Biochem. Pharmacol. 98: 63-175.

Frankenhaeuser, B., and A. L. Hodgkin (1956) The after effects of impulses in the giant nerve fiber of Loligo. J. Physiol. (Lond.) 131: 341-376.

Greene, D. A., and A. I. Winegrad (1981) Effects of acute experimentation diabetes on composite energy metabolism in peripheral nerve axons and schwann cells. Diabetes 30:967.

Greene, D. A., S. Lattimer, J. Ulbrecht, and P. Carroll (1985) Glucose induced alteration in nerve metabolism: Current perspective on the pathogenesis of diabetic neuropathy and future direction for research and therapy. Diabetes Care 8: 290-299.

Greene, D. A., S. Lattimer, and A. A. F. Sima (1987) Sorbitol, phosphoinositides and sodium-potassium-ATPase in the pathogenesis of diabetic complications. N. Engl. J. Med. 316: 599-606.

Grossman, Y., and M. J. Gutnick (1981) Extracellular potassium activity during frequency dependent conduction block of giant axons in the metathoracic ganglion of the cockroach. Brain Res. 211: 196201.

Grossman, Y., I. Parnas, and M. E. Spira (1979a) Differential conduction block in branches of bifurcating axon. J. Physiol. (Lond.) 295: 283-305.

Grossman, Y., I. Parnas, and M. E. Spira (1979b) Ionic mechanisms of differential conduction of action potential at high frequency in branching axon. J. Physiol. (Lond.) 295: 307-322.

Harati, Y. (1987) Diabetic peripheral neuropathies. Ann. Int. Med. 107: 546-559.
Hatt, H., and D. O. Smith (1976) Synaptic depression related to presynaptic axon conduction block. J. Physiol. (Lond.) 259: 367-393.

Henning, R., and T. Lomo (1985) Firing patterns of motor units in normal rats. Nature $314:$ 164-166.

Horowitz, S. H., and H. Ginsberg-Fellner (1979) Ischaemia and sensory nerve conduction in diabetes mellitus. Neurology 29: 695-704.

Jakobsen, J. (1976) Axonal dwindling in early experimental diabetes. I. Study of cross sectioned nerves. Diabetologia 12: 539-546.

Jakobsen, J. (1978) Peripheral nerve in early experimental diabetes. Diabetologia 14: 113-119.

Kennedy, W. K., U. J. Quick, T. Miyoshi, and G. C. Gerritsen (1982) Peripheral neuropathy of diabetic chinese hamster. Diabetologia 23: 445-451.

Kocsis, J. D., R. C. Malenka, and S. G. Waxman (1983) Effects of extracellular potassium concentration on the excitability of the parallel fibers (PFS) of the rat cerebellum. J. Physiol. (Lond.) 334: $225-$ 244.

Krnjevic, K., and R. Miledi (1958) Failure of neuromuscular propagation in rats. J. Physiol. (Lond.) 140: 440-461.

Krnjevic, K., and R. Miledi (1959) Presynaptic failure of neuromuscular propagation. J. Physiol. (Lond.) 149: 1-22.

Lev-Tov, A., and R. Rahamimoff (1980) A study of tetanic and post tetanic potentiation of miniature end plate potentials at the frog neuromuscular junction. J. Physiol. (Lond.) 309: 247-273.

Low, A. P. (1987) Recent advances in the pathogenesis of diabetic neuropathy. Muscle Nerve 10: 121-128.

Low, P. A., K. Waro, J. D. Schmelzer, and S. Brimjoin (1985) Ischaemic conduction failure and energy metabolism in experimental diabetic neuropathy. Am. J. Physiol. 248: E457-462.

Magleby, K. L., and J. E. Zengel (1975) A quantative description of tetanic and post tetanic potentiation of transmitter release at the frog neuromuscular junction. J. Physiol. (Lond.) 245: 183-208.

Martinez, A.C. (1986) Diabetic neuropathy: Topography, general electrophysiological features and effect of ischaemia on nerve evoked potentials. Electromyogr. Clin. Neurophysiol. 26: 283-295.

Mulder, D. W., E. A. Lambert, J. A. Bastron, and R. G. Sprague (1961) The neuropathies associated with diabetes mellitus. Neurology 11 : 275-284.

Nicoll, R. A. (1979) Dorsal root potentials and changes in extracellular potassium in spinal cord of the frog. J. Physiol. (Lond.) 290: 113 127.

Nielsen, V. K. (1982) Mechanisms of preserved nerve function with ischaemia in peripheral neuropathy. Kyoto Symp. 36: 70-80.

Nussinovitch, I., and R. Rahamimoff (1988) Ionic basis of tetanic and post tetanic potentiation at a mammalian neuromuscular junction. $\mathrm{J}$. Physiol. (Lond.) 396: 435-455.

Parnas, I., and I. Segev (1979) A mathematical model for conduction of action potentials along bifurcating axons. J. Physiol. (Lond.) 295: 323-343.

Pirart, J. (1978) Diabetes mellitus and degenerative complications: A prospective study of 4400 patients observed between 1947 and 1973 . Diabetes Care 1: 168-188, 252-263.

Rall, W. (1962) Theory of physiological properties in dendrites. Ann. NY Acad. Sci. 96: 1071-1092.

Robertson, D. M., and A. A. F. Sima (1980) Diabetic neuropathy in mutant mouse. A morphometric study. Diabetes 29:60-67.

Senevirante, K. N., and O. A. Peiris (1970) The role of diffusion barriers in determining the excitability of peripheral nerve. J. Neurol. Neurosurg. Psychiatry 33: 310-318.

Shirabe, S., I. Kinoshita, H. Matsuo, H. Takashima, T. Nakamura, M. Tsujihata, and S. Nagataki (1988) Resistance to ischemic conduction block of the peripheral nerve in hyperglycemic rats: An electrophysiological study. Muscle Nerve 11: 582-587.

Sima, A. A. F., and M. C. Hay (1981) Consideration of the neuropathy in the diabetic BB Wistar rat. Neuropathol. Appl. Neurobiol. 7:341350.

Sjogren, A., C. H. Floren, and A. Nilsson (1986) Magnesium deficiency in IDDM related to level of glycosylated haemoglobin. Diabetes 35: 459-463.

Smith, D. O. (1979) Capability of synaptic transmission in aged rats. Exp. Neurol. 66: 650-666.

Smith, D. O. (1980) Mechanism of action potential propagation failure at sites of axon branching in the crayfish. J. Physiol. (Lond.) 301: 243-259.

Smith, D. O. (1983) Extracellular potassium levels and axon excit- 
ability during repetitive action potential in crayfish. J. Physiol. (Lond.) 336: 143-158.

Somjen, G. S. (1979) Extracellular potassium in the mammalian central nervous system. Annu. Rev. Physiol. 41: 159-177.

Spira, M. E., Y. Yarom, and I. Parnas (1976) Modulation of spike frequency by regions of special axonal geometry and by synaptic output. J. Neurophysiol. 39: 882-899.

Steiness, I. (1959) Vibratory perception in diabetics during arrested blood flow to the limb. Acta Med. Scand. 163: 195-205.

Tackmann, W., H. E. Kaeser, W. Berger, and A. N. Rueger (1981) Sensory and motor parameters in leg nerve of diabetes. Eur. Neurol. 20: 344-350.
Thomas, P. K., and S. G. Eliasson (1984) Diabetic neuropathy. In Peripheral Neuropathy, 2nd ed., P. J. Dyck, P. K. Thomas, E. H. Lambert, and R. P. Bunge, eds., pp. 1773-1810, W. B. Saunders, Philadelphia.

Van Essen, D. C. (1973) The contribution of membrane hyperpolarization to adaptation and conduction block in sensory neurons of the leech. J. Physiol. (Lond.) 230: 509-534.

Winegrad, A. I. (1987) Does a common mechanism induce the diverse complications of diabetes? Diabetes 36: 396-406. 